QUARTERLY OF APPLIED MATHEMATICS

VOLUME LXVII, NUMBER 4

DECEMBER 2009, PAGES 707-723

S 0033-569X(09)01149-0

Article electronically published on May 12, 2009

\title{
ON SPATIAL BEHAVIOR IN LINEAR VISCOELASTICITY
}

\author{
BY \\ CĂTĂLIN GALEŞ (Faculty of Mathematics, Al. I. Cuza University of Iaşi, Blvd. Carol I, no. 11, \\ 700506 - Iaşi, Romania) \\ AND \\ STAN CHIRIŢĂ (Faculty of Mathematics, Al. I. Cuza University of Iaşi, Blvd. Carol I, no. 11, \\ 700506 - Iaşi, Romania)
}

\begin{abstract}
Within the framework of linear viscoelasticity this paper deals with the study of spatial behavior of solutions describing harmonic vibrations in a right cylinder of finite extent. Some exponential decay estimates of Saint-Venant type, in terms of the distance from the excited end of the cylinder are obtained from a first-order differential inequality concerning an appropriate measure associated with the amplitude of the steady-state vibration. The dissipative mechanism guarantees the validity of the result for every value of the frequency of vibration and for the class of viscoelastic materials compatible with thermodynamics whose relaxation tensor is supposed to be symmetric and sufficiently regular. The case of a semi-infinite cylinder is also studied, and some alternatives of Phragmén-Lindelöf type are established.
\end{abstract}

Introduction. The present paper is concerned with the study of the spatial behavior of solutions in a right cylinder made of an anisotropic and homogeneous viscoelastic solid. We consider a finite cylinder subject to boundary data varying harmonically in time on one end, while the other end and lateral surface are clamped. The history of the displacement up to time $t=0$ is supposed to be known and the body force is assumed to be absent.

Initial boundary value problems of this type have been treated by Flavin and Knops [1] in the framework of the linearly damped wave equation and the linearly elastic damped cylinder. They proved that in both cases the existence of damping gives rise ultimately to a steady-state oscillation, whose amplitude decays exponentially from the excited end provided the exciting frequency is less than a certain critical value. The latter case has

Received May 16, 2008.

2000 Mathematics Subject Classification. Primary 74D05, 74G50; Secondary 74H45, 74E10.

Key words and phrases. Viscoelastic cylinder, harmonic vibrations, spatial behavior, dissipative effects. The authors are very grateful to the reviewer for useful observations which have led to the improvement of this paper. The authors were supported by the Romanian Ministry of Education and Research, CNCSIS Grant code ID-401, Contract no. 15/28.09.2007.

E-mail address: cgales@uaic.ro

E-mail address: schirita@uaic.ro 
been investigated under the assumption of positive definiteness upon the elasticity tensor. This work has been followed by further developments (see [2, 3, 4, 5, 6] and the references therein). The results established in these papers suggest exponential decay of activity away from the excited end, provided the frequency of vibration is lower than a critical value and the constitutive coefficients satisfy some positive definiteness conditions.

The present paper is devoted to the linear theory of viscoelasticity. We recall that mathematical theories of materials with fading memory have been developed to a great extent in recent decades (see, for example, the books by Day [7, Leitman and Fischer [8], Christensen [9] and Fabrizio and Morro [10]). Here, we consider a viscoelastic solid whose relaxation tensor is symmetric, sufficiently regular and compatible with thermodynamics. According to [10, 11], the compatibility with thermodynamics implies that $\dot{G}_{r l m n}^{s}(\cdot)$, the half-range Fourier sine transform of the relaxation tensor, is negative definite.

A prismatic cylinder made of a linear viscoelastic material is subject to a harmonic-intime vibration on its basis, while the remaining boundary is clamped. For the amplitude of the steady-state vibrations occurring in the cylinder we associate an adequate measure. Then, following standard arguments concerning the Saint-Venant's principle [12, 13, 1] we infer that the decay of the amplitude of vibrations is described by an exponential of the distance from the excited end of the cylinder, provided that the negative definiteness of $\dot{G}_{r l m n}^{s}(\cdot)$ is assumed. We also indicate how to extend the results to a semi-infinite cylinder.

Furthermore, we relax the assumption on the relaxation tensor by assuming that $-\dot{G}_{r l m n}^{s}(\cdot)$ is strongly elliptic. By introducing such a hypothesis we are considering a very large class of materials, including those new materials with extreme and unusual physical properties such as a negative Poisson's ratio (that is, so-called auxetic materials). These are materials with heterogeneous structure, including natural viscoelastic composites such as bone, ligament, and wood, as well as synthetic composites, biomaterials, and cellular solids with structural hierarchy (see, for example, [14, [15]). Such a material expands laterally when stretched, in contrast to ordinary materials. On the basis of the strong ellipticity of $-\dot{G}_{r l m n}^{s}(\cdot)$ we are able to introduce adequate measures of the amplitude of vibration and to establish appropriate spatial estimates describing the spatial evolution of the amplitude in question.

All spatial estimates established in the present paper hold for arbitrary values of the exciting frequency under the hypotheses upon the relaxation tensor suggested by the dissipation inequality. We recall that the spatial behavior of the transient component of the solution has been established by Chiriţă et al. 16] and Chiriţă and Ciarletta [17.

1. Formulation of the problem. Throughout this paper, we shall denote by $x_{1}$, $x_{2}, x_{3}$ the rectangular Cartesian coordinates and by $t$ the time. We employ the usual summation and differentiation conventions: Latin subscripts are understood to range over the integers $(1,2,3)$, whereas Greek subscripts are confined to the range $(1,2)$, summation over repeated subscripts is implied, subscripts preceded by a comma denote partial differentiation with respect to the corresponding Cartesian coordinate, and a superposed dot denotes time differentiation. 
We assume that $B$ is the interior of a right cylinder of length $L>0$ whose cross section is bounded by one or more piecewise smooth curves and choose the Cartesian coordinates such that the origin lies in the excited end of the cylinder and such that the $x_{3}$-axis is parallel to the generators. Let $D\left(x_{3}\right)$ denote the cross section of the cylinder corresponding to the axial distance $x_{3}$ to the excited end, and let $\partial D\left(x_{3}\right)$ denote the cross-sectional boundary. We denote by $\pi$ the lateral surface of the cylinder, that is, $\pi=\partial D \times[0, L]$.

Let us suppose that a homogeneous and anisotropic linear viscoelastic material fills $B$. According to the linearized theory of isothermal viscoelasticity [10, the fundamental system of field equations, in the absence of the body force, consists of the strain-displacement relations

$$
e_{r l}=\frac{1}{2}\left(u_{r, l}+u_{l, r}\right), \quad \text { in } B \times(-\infty, \infty),
$$

the constitutive equations

$$
t_{r l}=G_{r l m n}(0) e_{m n}+\int_{0}^{\infty} \dot{G}_{r l m n}(s) e_{m n}^{t}(s) d s, \quad \text { in } B \times[0, \infty),
$$

and the equations of motion

$$
t_{r l, r}=\rho_{0} \ddot{u}_{l}, \quad \text { in } B \times(0, \infty),
$$

where $u_{l}$ are the components of the displacement vector, $e_{r l}$ are the components of the strain tensor, $t_{r l}$ are the components of the stress tensor and $e_{r l}^{t}$ represent the history up to time $t$, namely $e_{r l}^{t}(s)=e_{r l}(t-s), s \geq 0$. Further, $\rho_{0}$ is the constant mass density and $G_{r l m n}(t), 0 \leq t<\infty$, are the components of the relaxation tensor satisfying the symmetry relations

$$
G_{r l m n}(t)=G_{l r m n}(t)=G_{r l n m}(t), \quad t \geq 0 .
$$

The tensor $G_{r t m n}(0)$ is called the instantaneous elastic modulus and governs the response to instantaneous changes in the strain. We suppose that the relaxation tensor $G_{r l m n}(\cdot)$ has a continuous derivative $\dot{G}_{r l m n}(\cdot)$ and that the equilibrium elastic modulus

$$
G_{r l m n}(\infty)=\lim _{t \rightarrow \infty} G_{r l m n}(t)
$$

exists. We take $G_{r l m n}(\infty)$ to be a positive definite tensor so that the body is a solid. Moreover, we assume that the relaxation tensor is symmetric, that is,

$$
G_{r l m n}(t)=G_{m n r l}(t), \quad t \geq 0 .
$$

We recall that the behavior of the relaxation tensor $G_{r l m n}(t), 0 \leq t<\infty$, when the material is compatible with thermodynamics or the material is dissipative, has been discussed in various papers (see Day [18, Fabrizio and Morro [10, 11], Wilkes [19] and the references therein).

In this paper we suppose that the material is compatible with thermodynamics. According to Fabrizio and Morro [10, p. 47], this hypothesis assures that the half-range Fourier sine transform

$$
\dot{G}_{r l m n}^{s}(\omega)=\int_{0}^{\infty} \dot{G}_{r l m n}(s) \sin (\omega s) d s, \quad \omega>0,
$$


of the function $\dot{G}_{r l m n} \in L^{1}([0, \infty))$ is negative definite. Such a condition inspires the assumptions upon the relaxation tensor made in the present paper in order to establish the spatial behavior of the harmonic vibrations. In fact, the results described in Section 2 are obtained by assuming the positive definiteness of $-\dot{G}_{r l m n}^{s}(\omega)$, while in Section 3 we relax this condition by assuming $-\dot{G}_{r l m n}^{s}(\omega)$ to be strongly elliptic.

Since the above assumptions upon $\dot{G}_{r l m n}^{s}(\omega)$ there seem to be not so transparent, we illustrate the relationship between the relaxation tensor $G_{r l m n}(t)$ and the half-range Fourier sine transform $\dot{G}_{r l m n}^{s}(\omega)$ of $\dot{G}_{r l m n}(t)$ by a simple example. Thus, let us consider the case of decay exponential memory with $G_{r l m n}(t)=G_{r l m n}^{\infty}+e^{-\alpha t} H_{r l m n}, \alpha>0, H_{r l m n}$ and $G_{r l m n}^{\infty}$ independent of $t$ and $G_{r l m n}^{\infty}$ positive definite (the body is a solid). Then, the half-range Fourier sine transform is $\dot{G}_{r l m n}^{s}(\omega)=-\frac{\alpha \omega}{\alpha^{2}+\omega^{2}} H_{r l m n}$. Therefore, the half-range Fourier sine transform is negative definite if and only if $H_{r l m n}$ is positive definite, while the strong ellipticity of $-\dot{G}_{r l m n}^{s}(\omega)$ means the strong ellipticity of $H_{r l m n}$ and conversely. Particularly, in the isotropic case when $H_{r l m n}=\lambda \delta_{r l} \delta_{m n}+\mu\left(\delta_{r m} \delta_{l n}+\delta_{r n} \delta_{l m}\right)$, the positive definiteness of $-\dot{G}_{r l m n}^{s}(\omega)$ is equivalent with $\mu>0, \lambda+\frac{2}{3} \mu>0$, while the strong ellipticity is equivalent with $\mu>0, \lambda+2 \mu>0$ (see Gurtin [20] for a discussion concerning the strong ellipticity of the elastic tensor).

The situation changes a little when $G_{r l m n}(t)$ is a sum of exponential functions, that is, $G_{r l m n}(t)=G_{r l m n}^{\infty}+\sum_{P=1}^{N} e^{-\alpha_{P} t} H_{r l m n}^{P}, \alpha_{P}>0, G_{r l m n}^{\infty}$ and $H_{r l m n}^{P}$ independent of $t$ for all $P=1,2, \ldots, N$ and $G_{r l m n}^{\infty}$ positive definite. Clearly, $-\dot{G}_{r l m n}^{s}(\omega)$ is positive definite (or strongly elliptic) if and only if the linear combination $\sum_{P=1}^{N} \frac{\alpha_{P}}{\alpha_{P}^{2}+\omega^{2}} H_{r l m n}^{P}$ is positive definite (or strongly elliptic), so that it is not necessary that all tensors $H_{r l m n}^{P}$ be positive definite (or strongly elliptic) such that $-\dot{G}_{r l m n}^{s}(\omega)$ be positive definite (or strongly elliptic) but just a linear combination of the tensors $H_{r l m n}^{P}$.

Let us consider the Cauchy problem expressed by the relations (1.1), (1.2), (1.3), the lateral boundary conditions

$$
u_{l}=0, \quad \text { on } \pi \times[0, \infty)
$$

together with the end boundary conditions

$$
\begin{aligned}
& u_{l}=\tilde{u}_{l}\left(x_{1}, x_{2}\right) \exp (i \omega t), \quad \text { on } D(0) \times(0, \infty), \\
& u_{l}=0, \quad \text { on } D(L) \times(0, \infty)
\end{aligned}
$$

and the initial history condition of the displacement

$$
u_{l}=a_{l}\left(x_{1}, x_{2}, x_{3}, t\right), \quad \text { in } B \times(-\infty, 0] .
$$

In the above relations $\omega$ is a positive constant (frequency of vibration), $i=\sqrt{-1}$ is the unit complex number and $\tilde{u}_{l}, a_{l}$ are prescribed functions.

It is easy to see that

$$
u_{l}=U_{l}\left(x_{1}, x_{2}, x_{3}, t\right)+v_{l}\left(x_{1}, x_{2}, x_{3}\right) \exp (i \omega t),
$$

where $U_{l}$ (transient solution) absorbs the initial history and satisfies the null boundary conditions and the equations (1.1), (1.2) and (1.3), while $v_{l}$ (amplitude of steady-state 
solution) satisfies the boundary value problem consisting of the field equations

$$
\begin{aligned}
& T_{r l, r}=-\rho_{0} \omega^{2} v_{l}, \\
& T_{r l}=G_{r l m n}(0) \varepsilon_{m n}+\left(\int_{0}^{\infty} \dot{G}_{r l m n}(s) e^{-i \omega s} d s\right) \varepsilon_{m n}, \\
& \varepsilon_{r l}=\frac{1}{2}\left(v_{r, l}+v_{l, r}\right), \quad \text { in } B,
\end{aligned}
$$

subject to

$$
v_{l}=0, \quad \text { on } \pi
$$

and

$$
\begin{aligned}
& v_{l}=\tilde{u}_{l}\left(x_{1}, x_{2}\right), \quad \text { on } D(0), \\
& v_{l}=0, \quad \text { on } D(L) .
\end{aligned}
$$

If we introduce the notation

$$
C_{r l m n}(\omega)=G_{r l m n}(0)+\dot{G}_{r l m n}^{c}(\omega),
$$

where $\dot{G}_{r l m n}^{c}(\omega)$ is the half-range Fourier cosine transform of the function $\dot{G}_{r l m n} \in$ $L^{1}([0, \infty))$, i.e.

$$
\dot{G}_{r l m n}^{c}(\omega)=\int_{0}^{\infty} \dot{G}_{r l m n}(s) \cos (\omega s) d s,
$$

then from (1.7) and (1.13) we deduce the following system of partial differential equations:

$$
\left(C_{r l m n}(\omega)-i \dot{G}_{r l m n}^{s}(\omega)\right) v_{n, m r}+\rho_{0} \omega^{2} v_{l}=0 .
$$

For later convenience, we note that $T_{r l}$ may be written in the form

$$
T_{r l}=\left(C_{r l m n}(\omega)-i \dot{G}_{r l m n}^{s}(\omega)\right) \varepsilon_{m n} .
$$

$U_{l}$ represents essentially the transient, and $v_{l} \exp (i \omega t)$ is the forced oscillation. We recall that the spatial behavior of the transient solution $U_{l}$ can be studied by the methods developed by Chiriţă et al. [16] and Chiriţă and Ciarletta [17. So in what follows we will study the spatial behavior of the amplitude of the steady-state vibration satisfying (1.13) under the boundary conditions (1.14), (1.15) and (1.16). Since the effects caused by $U_{l}$ in the cylinder are transient, the decay estimates for $v_{l}$ are of interest for large time $t$.

2. Spatial behavior based on the positive definiteness of $-\dot{G}_{r l m n}^{s}(\omega)$. Throughout this section we shall assume that $-\dot{G}_{r l m n}^{s}(\omega)$ is positive definite. Let us consider the boundary value problem defined by the equations (1.19) (or (1.13)) and the boundary conditions (1.14), (1.15), (1.16) and let us introduce the following cross-sectional functional

$$
\begin{aligned}
& I\left(x_{3}\right)=\int_{D\left(x_{3}\right)} i\left(T_{r 3} \bar{v}_{r}-\bar{T}_{r 3} v_{r}\right) d A \\
& =\int_{D\left(x_{3}\right)}\left[i C_{r 3 m l}(\omega)\left(v_{l, m} \bar{v}_{r}-\bar{v}_{l, m} v_{r}\right)+\dot{G}_{r 3 m l}^{s}(\omega)\left(v_{l, m} \bar{v}_{r}+\bar{v}_{l, m} v_{r}\right)\right] d A,
\end{aligned}
$$


where the superposed bar denotes the complex conjugate. By using the symmetry assumption of $G_{r l m n}$ and the negative definiteness property of the tensor $\dot{G}_{r l m n}^{s}(\omega)$, we prove in this section that $I(\cdot)$ is an acceptable measure of the amplitude of steady-state vibrations that decay more rapidly than an exponential of the distance from the excited end of the cylinder. To this end we follow the standard procedures concerning the Saint-Venant's principle [12, 13, 1].

Thus, from (2.1), by direct differentiation, we get

$$
\begin{aligned}
& \frac{d I}{d x_{3}}\left(x_{3}\right)=\int_{D\left(x_{3}\right)}\left[i C_{r 3 m l}(\omega)\left(v_{l, m} \bar{v}_{r, 3}-\bar{v}_{l, m} v_{r, 3}\right)+\dot{G}_{r 3 m l}^{s}(\omega)\left(v_{l, m} \bar{v}_{r, 3}+\bar{v}_{l, m} v_{r, 3}\right)\right] d A \\
& +\int_{D\left(x_{3}\right)}\left[i C_{r 3 m l}(\omega)\left(v_{l, m 3} \bar{v}_{r}-\bar{v}_{l, m 3} v_{r}\right)+\dot{G}_{r 3 m l}^{s}(\omega)\left(v_{l, m 3} \bar{v}_{r}+\bar{v}_{l, m 3} v_{r}\right)\right] d A .
\end{aligned}
$$

By using the field equations (1.19), the square bracket of the second integral may be written in the form

$$
\begin{aligned}
& i C_{r 3 m l}(\omega)\left(v_{l, m 3} \bar{v}_{r}-\bar{v}_{l, m 3} v_{r}\right)+\dot{G}_{r 3 m l}^{s}(\omega)\left(v_{l, m 3} \bar{v}_{r}+\bar{v}_{l, m 3} v_{r}\right) \\
& =-i C_{r \rho m l}(\omega)\left(v_{l, m \rho} \bar{v}_{r}-\bar{v}_{l, m \rho} v_{r}\right)-\dot{G}_{r \rho m l}^{s}(\omega)\left(v_{l, m \rho} \bar{v}_{r}+\bar{v}_{l, m \rho} v_{r}\right) .
\end{aligned}
$$

The divergence theorem, the boundary conditions (1.14), the symmetry of the tensors $C_{r n m l}(\omega), \dot{G}_{r n m l}^{s}(\omega)$ and the above relation may be used to write $d I / d x_{3}$ in the form

$$
\frac{d I}{d x_{3}}\left(x_{3}\right)=2 \int_{D\left(x_{3}\right)} \dot{G}_{r n m l}^{s}(\omega) v_{l, m} \bar{v}_{r, n} d A .
$$

Now, since the fourth-order tensor $-\dot{\mathbf{G}}^{s}(\omega)$ is symmetric and positive definite, then there exist two positive constants depending on $\omega$, denoted by $\nu^{m}$ and $\nu^{M}$, such that

$$
\nu^{m}(\omega) \zeta_{l r} \bar{\zeta}_{l r} \leq-\dot{G}_{l r n p}^{s}(\omega) \zeta_{l r} \bar{\zeta}_{n p} \leq \nu^{M}(\omega) \zeta_{l r} \bar{\zeta}_{l r},
$$

for every symmetric second-order tensor $\zeta_{l r}$. From (2.4) and (2.5) we deduce

$$
\frac{d I}{d x_{3}}\left(x_{3}\right) \leq-2 \nu^{m}(\omega) \int_{D\left(x_{3}\right)} \varepsilon_{l r} \bar{\varepsilon}_{l r} d A \leq 0
$$

where $\varepsilon_{l r}$ is defined by $(1.13)_{3}$, so that $I(\cdot)$ is a nonincreasing function. We also note that (1.16) assures that $I(L)=0$, so that $I\left(x_{3}\right) \geq 0$ for all $x_{3} \in[0, L]$.

On the other hand, letting $\sigma$ be a positive parameter and using Schwarz and arithmeticgeometric mean inequalities one deduces

$$
I\left(x_{3}\right) \leq \int_{D\left(x_{3}\right)}\left|T_{r 3} \overline{i v}_{r}+\bar{T}_{r 3} i v_{r}\right| d A \leq \sigma \int_{D\left(x_{3}\right)} T_{r l} \bar{T}_{r l} d A+\frac{1}{\sigma} \int_{D\left(x_{3}\right)} v_{r} \bar{v}_{r} d A .
$$

Denoting by $\Upsilon^{\prime}$ and $\Upsilon^{\prime \prime}$ the real and imaginary parts of the complex quantity $\Upsilon$, then from $(1.13) 3$ and (1.20) it follows that

$$
\begin{aligned}
& T_{r l}^{\prime}=C_{r l m n}(\omega) \varepsilon_{m n}^{\prime}+\dot{G}_{r l m n}^{s}(\omega) \varepsilon_{m n}^{\prime \prime}, \\
& T_{r l}^{\prime \prime}=C_{r l m n}(\omega) \varepsilon_{m n}^{\prime \prime}-\dot{G}_{r l m n}^{s}(\omega) \varepsilon_{m n}^{\prime} .
\end{aligned}
$$


Let us denote by $c_{M}^{2}(\omega)$ the largest eigenvalue of the symmetric and positive semidefinite tensor $B_{\text {snpq }}(\omega)=C_{r l s n}(\omega) C_{r l p q}(\omega)$. Then by the usual techniques it may be established that

$$
T_{r l} \bar{T}_{r l}=T_{r l}^{\prime} T_{r l}^{\prime}+T_{r l}^{\prime \prime} T_{r l}^{\prime \prime} \leq\left(c_{M}(\omega)+\nu^{M}(\omega)\right)^{2} \varepsilon_{r l} \bar{\varepsilon}_{r l} .
$$

Further, letting $B(l, h)$ denote the cylinder slice bounded by the plane ends $x_{3}=l$ and $x_{3}=l+h$, then the boundary condition (1.14) and the relation (2.5) assure that the following inequalities hold:

$$
\begin{aligned}
& -\int_{B\left(x_{3}, h\right)} \dot{G}_{r n m l}^{s}(\omega) \varepsilon_{r n}^{\prime} \varepsilon_{m l}^{\prime} d V \geq \rho_{0} w_{0}^{2}(h) \int_{B\left(x_{3}, h\right)} v_{r}^{\prime} v_{r}^{\prime} d V, \\
& -\int_{B\left(x_{3}, h\right)} \dot{G}_{r n m l}^{s}(\omega) \varepsilon_{r n}^{\prime \prime} \varepsilon_{m l}^{\prime \prime} d V \geq \rho_{0} w_{0}^{2}(h) \int_{B\left(x_{3}, h\right)} v_{r}^{\prime \prime} v_{r}^{\prime \prime} d V,
\end{aligned}
$$

where $\frac{w_{0}(h)}{2 \pi}$ is the lowest frequency of vibration of the cylinder $B\left(x_{3}, h\right)$ filled by an elastic material whose components of the constant elasticity tensor are $-\dot{G}_{r n m l}^{s}(\omega)$ and whose lateral surface is clamped and whose plane ends are free. We note that more information concerning the inequalities (2.10) and the frequency $w_{0}(h)$ may be found in the paper by Toupin [12] and in the monograph article by Gurtin [20].

From (2.10) we deduce the following Poincaré-type inequality:

$$
-\int_{B\left(x_{3}, h\right)} \dot{G}_{r n m l}^{s}(\omega) \varepsilon_{r n} \bar{\varepsilon}_{m l} d V \geq \rho_{0} w_{0}^{2}(h) \int_{B\left(x_{3}, h\right)} v_{r} \bar{v}_{r} d V .
$$

The relations (2.5), (2.7), (2.9) and (2.11) assure that the function

$$
Q\left(x_{3}, h\right)=\frac{1}{h} \int_{x_{3}}^{x_{3}+h} I(y) d y
$$

satisfies the inequality

$$
Q\left(x_{3}, h\right) \leq\left(\frac{\sigma\left(c_{M}(\omega)+\nu^{M}(\omega)\right)^{2}}{h}+\frac{\nu^{M}(\omega)}{\sigma h \rho_{0} w_{0}^{2}(h)}\right) \int_{B\left(x_{3}, h\right)} \varepsilon_{r l} \bar{\varepsilon}_{r l} d V .
$$

On integrating (2.6) between $x_{3}$ and $x_{3}+h$ and using the definition (2.12) we deduce

$$
\frac{\partial Q}{\partial x_{3}}\left(x_{3}, h\right)=\frac{1}{h}\left(I\left(x_{3}+h\right)-I\left(x_{3}\right)\right) \leq-\frac{2 \nu^{m}}{h} \int_{B\left(x_{3}, h\right)} \varepsilon_{r l} \bar{\varepsilon}_{r l} d V .
$$

Thus, from (2.13) and (2.14) one obtains

$$
\gamma(\sigma, h, \omega) \frac{\partial Q}{\partial x_{3}}\left(x_{3}, h\right)+Q\left(x_{3}, h\right) \leq 0,
$$

where

$$
\gamma(\sigma, h, \omega)=\frac{\sigma\left(c_{M}(\omega)+\nu^{M}(\omega)\right)^{2}}{2 \nu^{m}(\omega)}+\frac{\nu^{M}(\omega)}{2 \sigma \rho_{0} w_{0}^{2}(h) \nu^{m}(\omega)} .
$$


Setting the parameter $\sigma$ in order that the above quantity be a minimum, that is,

$$
\begin{aligned}
& \sigma_{0}=\frac{1}{c_{M}(\omega)+\nu^{M}(\omega)} \sqrt{\frac{\nu^{M}(\omega)}{\rho_{0} w_{0}^{2}(h)}}, \\
& \gamma_{m}(h, \omega)=\gamma\left(\sigma_{0}, h, \omega\right)=\frac{c_{M}(\omega)+\nu^{M}(\omega)}{\nu^{m}(\omega)} \sqrt{\frac{\nu^{M}(\omega)}{\rho_{0} w_{0}^{2}(h)}}
\end{aligned}
$$

from (2.15) we deduce

$$
Q\left(x_{3}, h\right) \leq Q(0, h) \exp \left(-\frac{x_{3}}{\gamma_{m}(h, \omega)}\right) .
$$

Moreover, since $I(\cdot)$ is a nonincreasing function on $[0, L]$, we have

$$
I\left(x_{3}+h\right) \leq Q\left(x_{3}, h\right) \leq I\left(x_{3}\right) .
$$

The above relations lead to the following.

Theorem 2.1. The cross-sectional function $I\left(x_{3}\right)$ defined by (2.1) in connection with the boundary value problem (1.13)-(1.16) is an acceptable measure of solution and satisfies the following decay estimate:

$$
0 \leq I\left(x_{3}\right) \leq I(0) \exp \left(-\frac{x_{3}-h}{\gamma_{m}(h, \omega)}\right), \quad h \leq x_{3} \leq L,
$$

where $\gamma_{m}(h, \omega)$ is defined by (2.17) 2 .

3. Spatial behavior under the strong ellipticity of $-\dot{G}_{r l m n}^{s}(\omega)$. In this section we will study the spatial behavior of the amplitude of vibrations by using the strong ellipticity condition for $-\dot{G}_{r l m n}^{s}(\omega)$, that is,

$$
-\dot{G}_{r l m n}^{s}(\omega) \xi_{r} \xi_{m} \zeta_{l} \zeta_{n}>0
$$

for all nonzero vectors $\left(\xi_{1}, \xi_{2}, \xi_{3}\right),\left(\zeta_{1}, \zeta_{2}, \zeta_{3}\right)$. Such a condition is obviously milder than that expressing the positive definiteness of $-\dot{G}_{r l m n}^{s}(\omega)$. In fact, by using this hypothesis we are considering a very large class of materials including those new materials with extreme and unusual physical properties such as a negative Poisson's ratio (that is, socalled auxetic materials). These are materials with heterogeneous structure, including natural viscoelastic composites such as bone, ligament, and wood, as well as synthetic composites, biomaterials, and cellular solids with structural hierarchy (see, for example, [14, [15]). Such a material expands laterally when stretched, in contrast to ordinary materials.

Since such a study can be too complex for general anisotropic viscoelastic materials, we proceed here to pursue such a procedure for some particularly important classes of materials, namely that of transversely isotropic and rhombic systems. 
3.1. Transversely isotropic materials. Many natural and man-made materials are classified as transversely isotropic (or hexagonal). Such materials are characterized by the fact that one can find a line that allows a rotation of the material about it without changing its properties. The plane, which is perpendicular to this line (the axis of rotational symmetry) is called a plane of symmetry or plane of isotropy. A modern example for such a material are laminates made of randomly oriented chopped fibers that are in general placed in a certain plane. The effective material properties for a bundled structure have no profound direction in that plane, which then becomes a plane of symmetry. Hence, each plane that contains the axis of rotation is a plane of symmetry, and therefore, transversely isotropic materials admit an infinite number of symmetries.

The restrictions placed on the relaxation tensor by transverse isotropy imply the following conditions:

$$
\begin{aligned}
& G_{1123}=G_{1131}=G_{1112}=G_{2223}=G_{2231}=G_{2212}=0, \\
& G_{3323}=G_{3331}=G_{3312}=G_{2331}=G_{2312}=G_{3112}=0,
\end{aligned}
$$

corresponding to the direction of transverse isotropy coinciding with the $x_{3}$ coordinate axis. Moreover, if we set

$$
\begin{aligned}
& g_{11}=-\dot{G}_{1111}^{s}, \quad g_{22}=-\dot{G}_{2222}^{s}, \quad g_{33}=-\dot{G}_{3333}^{s}, \quad g_{12}=-\dot{G}_{1122}^{s}, \quad g_{23}=-\dot{G}_{2233}^{s}, \\
& g_{31}=-\dot{G}_{3311}^{s}, \quad g_{44}=-\dot{G}_{2323}^{s}, \quad g_{55}=-\dot{G}_{3131}^{s}, \quad g_{66}=-\dot{G}_{1212}^{s},
\end{aligned}
$$

then the only nonzero components $\dot{G}_{r l m n}^{s}$ are

$$
g_{22}=g_{11}, \quad g_{33}, \quad g_{23}=g_{13}, \quad g_{12}, \quad g_{44}=g_{55}, \quad g_{66}=\frac{1}{2}\left(g_{11}-g_{12}\right)
$$

and the strong ellipticity condition (3.1) becomes

$$
\begin{aligned}
& g_{66}\left(\xi_{1} \zeta_{2}-\xi_{2} \zeta_{1}\right)^{2}+g_{11}\left(\xi_{1} \zeta_{1}+\xi_{2} \zeta_{2}\right)^{2}+2\left(g_{13}+g_{55}\right)\left(\xi_{1} \zeta_{1}+\xi_{2} \zeta_{2}\right) \xi_{3} \zeta_{3} \\
& \quad+g_{33} \xi_{3}^{2} \zeta_{3}^{2}+g_{55}\left(\xi_{3}^{2} \zeta_{1}^{2}+\xi_{1}^{2} \zeta_{3}^{2}+\xi_{3}^{2} \zeta_{2}^{2}+\xi_{2}^{2} \zeta_{3}^{2}\right)>0 .
\end{aligned}
$$

As is well known (see, for example, Merodio and Ogden [21], Chiriţă 22]), such a condition holds if and only if

$$
\begin{aligned}
& g_{11}>0, \quad g_{33}>0, \quad g_{55}>0, \quad g_{11}>g_{12}, \\
& \left|g_{13}+g_{55}\right|<g_{55}+\sqrt{g_{11} g_{33}} .
\end{aligned}
$$

On the other hand, the differential system (1.19) becomes

$$
\begin{aligned}
& \left(c_{11}+i g_{11}\right) v_{1,11}+\left[c_{12}+c_{66}+i\left(g_{12}+g_{66}\right)\right] v_{2,21}+\left(c_{66}+i g_{66}\right) v_{1,22} \\
& +\left[c_{13}+c_{55}+i\left(g_{13}+g_{55}\right)\right] v_{3,31}+\left(c_{55}+i g_{55}\right) v_{1,33}+\rho_{0} \omega^{2} v_{1}=0, \\
& {\left[c_{12}+c_{66}+i\left(g_{12}+g_{66}\right)\right] v_{1,12}+\left(c_{66}+i g_{66}\right) v_{2,11}+\left(c_{11}+i g_{11}\right) v_{2,22}} \\
& \quad+\left[c_{13}+c_{55}+i\left(g_{13}+g_{55}\right)\right] v_{3,32}+\left(c_{55}+i g_{55}\right) v_{2,33}+\rho_{0} \omega^{2} v_{2}=0 \\
& \left(c_{55}+i g_{55}\right)\left(v_{3,11}+v_{3,22}\right)+\left[c_{13}+c_{55}+i\left(g_{13}+g_{55}\right)\right]\left(v_{1,13}+v_{2,23}\right) \\
& +\left(c_{33}+i g_{33}\right) v_{3,33}+\rho_{0} \omega^{2} v_{3}=0
\end{aligned}
$$


where

$$
c_{11}=C_{1111}, \quad c_{33}=C_{3333}, \quad c_{12}=C_{1122}, \quad c_{31}=C_{3311}, \quad c_{55}=C_{3131}, \quad c_{66}=C_{1212} .
$$

By means of the relations (1.14) and (3.8), we obtain the following identity:

$$
\begin{aligned}
& \frac{d}{d x_{3}} \int_{D\left(x_{3}\right)}\left\{i \left[\left(c_{13}+c_{55}\right)\left(v_{3} \bar{v}_{\rho, \rho}-\bar{v}_{3} v_{\rho, \rho}\right)+c_{55}\left(v_{\rho} \bar{v}_{\rho, 3}-\bar{v}_{\rho} v_{\rho, 3}\right)\right.\right. \\
& \left.\quad+c_{33}\left(v_{3} \bar{v}_{3,3}-\bar{v}_{3} v_{3,3}\right)\right]+g_{33}\left(v_{3} \bar{v}_{3,3}+\bar{v}_{3} v_{3,3}\right)+g_{55}\left(v_{\rho} \bar{v}_{\rho, 3}+\bar{v}_{\rho} v_{\rho, 3}\right) \\
& \left.\quad+\left(g_{13}+g_{55}\right)\left(v_{3} \bar{v}_{\rho, \rho}+\bar{v}_{3} v_{\rho, \rho}\right)\right\} d A=2 \int_{D\left(x_{3}\right)} g_{66}\left(v_{1,2}-v_{2,1}\right)\left(\bar{v}_{1,2}-\bar{v}_{2,1}\right) d A \\
& \quad+2 \int_{D\left(x_{3}\right)}\left[g_{11}\left(v_{1,1}+v_{2,2}\right)\left(\bar{v}_{1,1}+\bar{v}_{2,2}\right)+g_{33} v_{3,3} \bar{v}_{3,3}\right] d A \\
& \quad+2 \int_{D\left(x_{3}\right)} g_{55}\left(v_{1,3} \bar{v}_{1,3}+v_{3,1} \bar{v}_{3,1}+v_{2,3} \bar{v}_{2,3}+v_{3,2} \bar{v}_{3,2}\right) d A \\
& \quad+2 \int_{D\left(x_{3}\right)}\left(g_{13}+g_{55}\right)\left(v_{\rho, \rho} \bar{v}_{3,3}+\bar{v}_{\rho, \rho} v_{3,3}\right) d A
\end{aligned}
$$

Thus, we can introduce the function

$$
\begin{aligned}
& J_{\kappa}\left(x_{3}\right)=-\int_{D\left(x_{3}\right)}\left\{i \left[\left(c_{13}+c_{55}\right)\left(v_{3} \bar{v}_{\rho, \rho}-\bar{v}_{3} v_{\rho, \rho}\right)+c_{55}\left(v_{\rho} \bar{v}_{\rho, 3}-\bar{v}_{\rho} v_{\rho, 3}\right)\right.\right. \\
& \left.\quad+c_{33}\left(v_{3} \bar{v}_{3,3}-\bar{v}_{3} v_{3,3}\right)\right]+v_{\rho}\left[g_{55} \bar{v}_{\rho, 3}+\left(g_{55}-\kappa\right) \bar{v}_{3, \rho}\right]+\bar{v}_{\rho}\left[g_{55} v_{\rho, 3}+\left(g_{55}-\kappa\right) v_{3, \rho}\right] \\
& \left.\quad+v_{3}\left[g_{33} \bar{v}_{3,3}+\left(g_{13}+\kappa\right) \bar{v}_{\rho, \rho}\right]+\bar{v}_{3}\left[g_{33} v_{3,3}+\left(g_{13}+\kappa\right) v_{\rho, \rho}\right]\right\} d A,
\end{aligned}
$$

where $\kappa \in\left(0,2 g_{55}\right)$ is a positive parameter at our disposal. Then, the identity (3.10) when combined with (1.14) furnishes

$$
\begin{aligned}
& -\frac{d J_{\kappa}}{d x_{3}}\left(x_{3}\right)=2 \int_{D\left(x_{3}\right)} g_{66}\left(v_{1,2}-v_{2,1}\right)\left(\bar{v}_{1,2}-\bar{v}_{2,1}\right) d A \\
& \quad+2 \int_{D\left(x_{3}\right)}\left[g_{11} v_{\alpha, \alpha} \bar{v}_{\rho, \rho}+\left(g_{13}+\kappa\right)\left(v_{\rho, \rho} \bar{v}_{3,3}+\bar{v}_{\rho, \rho} v_{3,3}\right)+g_{33} v_{3,3} \bar{v}_{3,3}\right] d A \\
& \quad+2 \int_{D\left(x_{3}\right)}\left[g_{55}\left(v_{1,3} \bar{v}_{1,3}+v_{3,1} \bar{v}_{3,1}\right)+\left(g_{55}-\kappa\right)\left(v_{1,3} \bar{v}_{3,1}+\bar{v}_{1,3} v_{3,1}\right)\right] d A \\
& \quad+2 \int_{D\left(x_{3}\right)}\left[g_{55}\left(v_{2,3} \bar{v}_{2,3}+v_{3,2} \bar{v}_{3,2}\right)+\left(g_{55}-\kappa\right)\left(v_{2,3} \bar{v}_{3,2}+\bar{v}_{2,3} v_{3,2}\right)\right] d A .
\end{aligned}
$$

Further, for every $g_{13}$ satisfying (3.7) it is possible to determine $\kappa \in\left(0,2 g_{55}\right)$ so that

$$
\left|g_{13}+\kappa\right|<\sqrt{g_{11} g_{33}} .
$$

In fact, the choice for $\kappa$ has to be such that

$$
\max \left(-g_{13}-\sqrt{g_{11} g_{33}}, 0\right)<\kappa<\min \left(2 g_{55},-g_{13}+\sqrt{g_{11} g_{33}}\right) .
$$

Throughout the remainder of this section we assume that $\kappa$ satisfies the relation (3.14). With this choice we have

$$
\left|g_{55}-\kappa\right|<g_{55}
$$


and hence we can deduce that

$$
\begin{aligned}
& g_{55}\left(v_{1,3} \bar{v}_{1,3}+v_{3,1} \bar{v}_{3,1}\right)+\left(g_{55}-\kappa\right)\left(v_{1,3} \bar{v}_{3,1}+\bar{v}_{1,3} v_{3,1}\right) \\
& \quad \geq \eta_{1}\left(v_{1,3} \bar{v}_{1,3}+v_{3,1} \bar{v}_{3,1}\right), \\
& g_{55}\left(v_{2,3} \bar{v}_{2,3}+v_{3,2} \bar{v}_{3,2}\right)+\left(g_{55}-\kappa\right)\left(v_{2,3} \bar{v}_{3,2}+\bar{v}_{2,3} v_{3,2}\right) \\
& \quad \geq \eta_{1}\left(v_{2,3} \bar{v}_{2,3}+v_{3,2} \bar{v}_{3,2}\right),
\end{aligned}
$$

where

$$
\eta_{1}=\min \left(\kappa, 2 g_{55}-\kappa\right) .
$$

Moreover, the relation (3.13) implies that

$$
\begin{aligned}
& g_{11} v_{\alpha, \alpha} \bar{v}_{\rho, \rho}+\left(g_{13}+\kappa\right)\left(v_{\rho, \rho} \bar{v}_{3,3}+\bar{v}_{\rho, \rho} v_{3,3}\right)+g_{33} v_{3,3} \bar{v}_{3,3} \\
& \quad \geq \eta_{2}\left(v_{\alpha, \alpha} \bar{v}_{\rho, \rho}+v_{3,3} \bar{v}_{3,3}\right)
\end{aligned}
$$

where

$$
\eta_{2}=\frac{1}{2}\left[g_{11}+g_{33}-\sqrt{\left(g_{11}-g_{33}\right)^{2}+4\left(g_{13}+\kappa\right)^{2}}\right] .
$$

So we obtain

$$
\begin{aligned}
& -\frac{d J_{\kappa}}{d x_{3}}\left(x_{3}\right) \geq 2 \int_{D\left(x_{3}\right)} g_{66}\left(v_{1,2}-v_{2,1}\right)\left(\bar{v}_{1,2}-\bar{v}_{2,1}\right) d A \\
& \quad+2 \int_{D\left(x_{3}\right)} \eta_{2}\left(v_{\alpha, \alpha} \bar{v}_{\rho, \rho}+v_{3,3} \bar{v}_{3,3}\right) d A+2 \int_{D\left(x_{3}\right)} \eta_{1}\left(v_{\alpha, 3} \bar{v}_{\alpha, 3}+v_{3, \alpha} \bar{v}_{3, \alpha}\right) d A
\end{aligned}
$$

and

$$
-\frac{d J_{\kappa}}{d x_{3}}\left(x_{3}\right) \geq 2 \int_{D\left(x_{3}\right)} \eta_{1}\left(v_{\alpha, 3} \bar{v}_{\alpha, 3}+v_{3, \alpha} \bar{v}_{3, \alpha}\right) d A+2 \int_{D\left(x_{3}\right)}\left(\eta_{0} v_{\alpha, \beta} \bar{v}_{\alpha, \beta}+\eta_{2} v_{3,3} \bar{v}_{3,3}\right) d A,
$$

where

$$
\eta_{0}=\min \left(\eta_{2}, g_{66}\right) \text {. }
$$

Thus, from the relations (3.20) and (3.21) we can conclude that $\frac{d J_{\kappa}}{d x_{3}}\left(x_{3}\right) \leq 0$ and therefore $J_{\kappa}\left(x_{3}\right)$ is a nonincreasing function on $[0, L]$. Moreover, by recalling that the relations (1.14) and (3.11) imply $J_{\kappa}(L)=0$, it follows that $J_{\kappa}\left(x_{3}\right) \geq 0$ and hence it can be an acceptable measure for the amplitude of vibration.

Now we will proceed to obtain an appropriate estimate for the measure $J_{\kappa}\left(x_{3}\right)$. To this end we will use the lateral boundary condition (1.14) in order to write

$$
\int_{D\left(x_{3}\right)} v_{r, \alpha} \bar{v}_{r, \alpha} d A \geq \lambda_{0} \int_{D\left(x_{3}\right)} v_{r} \bar{v}_{r} d A,
$$

where $\lambda_{0}$ is the lowest eigenvalue in the two-dimensional clamped membrane problem for the cross section $D\left(x_{3}\right)$. Then, by means of the Schwarz and arithmetic-geometric mean 
inequalities, from (3.11), (3.13) and (3.15) we obtain

$$
\begin{aligned}
& J_{\kappa}\left(x_{3}\right) \leq \frac{1}{\sqrt{\lambda_{0}}}\left(\left|c_{13}+c_{55}\right|+\sqrt{g_{11} g_{33}}\right) \int_{D\left(x_{3}\right)} v_{\alpha, \alpha} \bar{v}_{\rho, \rho} d A \\
& +\frac{1}{\sqrt{\lambda_{0}}}\left(\left|c_{55}\right|+2 g_{55}\right) \int_{D\left(x_{3}\right)} v_{\alpha, \rho} \bar{v}_{\alpha, \rho} d A \\
& +\frac{1}{\sqrt{\lambda_{0}}}\left(\left|c_{13}+c_{55}\right|+\left|c_{33}\right|+g_{55}+g_{33}+\sqrt{g_{11} g_{33}}\right) \int_{D\left(x_{3}\right)} v_{3, \rho} \bar{v}_{3, \rho} d A \\
& +\frac{1}{\sqrt{\lambda_{0}}}\left(\left|c_{55}\right|+g_{55}\right) \int_{D\left(x_{3}\right)} v_{\alpha, 3} \bar{v}_{\alpha, 3} d A \\
& +\frac{1}{\sqrt{\lambda_{0}}}\left(\left|c_{33}\right|+g_{33}\right) \int_{D\left(x_{3}\right)} v_{3,3} \bar{v}_{3,3} d A .
\end{aligned}
$$

Furthermore, by using the relations (3.20) and (3.21) into (3.24), we obtain the following first-order differential inequality:

$$
J_{\kappa}\left(x_{3}\right) \leq-\eta \frac{d J_{\kappa}}{d x_{3}}\left(x_{3}\right) \quad \text { for all } x_{3} \in[0, L]
$$

where

$$
\begin{aligned}
\eta & =\frac{1}{2 \eta_{2} \sqrt{\lambda_{0}}}\left(\left|c_{13}+c_{55}\right|+\sqrt{g_{11} g_{33}}\right)+m_{0}, \\
m_{0} & =\frac{1}{2 \sqrt{\lambda_{0}}} \max \left(\frac{\left|c_{55}\right|+2 g_{55}}{\eta_{0}}, \frac{m_{1}}{\eta_{1}}, \frac{\left|c_{33}\right|+g_{33}}{\eta_{2}}\right), \\
m_{1} & =\max \left(\left|c_{55}\right|+g_{55},\left|c_{13}+c_{55}\right|+\left|c_{33}\right|+g_{55}+g_{33}+\sqrt{g_{11} g_{33}}\right) .
\end{aligned}
$$

By integrating the differential inequality (3.25) we can deduce the requested estimate. These results can be embodied in the following theorem.

Theorem 3.1. In the context of a finite cylinder made of a viscoelastic material, the cross-sectional functional $J_{\kappa}\left(x_{3}\right)$ represents an acceptable measure of the amplitude of vibration and satisfies the following exponential decay estimate:

$$
0 \leq J_{\kappa}\left(x_{3}\right) \leq J_{\kappa}(0) \exp \left(-\frac{x_{3}}{\eta}\right) \quad \text { for all } x_{3} \in[0, L] .
$$

3.2. Rhombic materials. In this subsection we shall consider the class of rhombic viscoelastic materials with the group $\mathcal{C}_{3}$ generated by $R_{\mathbf{e}_{3}}^{\pi}, R_{\mathbf{e}_{2}}^{\pi}$ (here $R_{\mathbf{e}}^{\theta}$ is the orthogonal tensor corresponding to a right-handed rotation through the angle $\theta, 0<\theta<2 \pi$, about an axis in the direction of the unit vector $e$ ). Such a class of viscoelastic materials is characterized by the only nonzero coefficients:

$$
\begin{aligned}
& g_{11}=-\dot{G}_{1111}^{s}, \quad g_{22}=-\dot{G}_{2222}^{s}, \quad g_{33}=-\dot{G}_{3333}^{s}, \quad g_{12}=-\dot{G}_{1122}^{s}, \quad g_{23}=-\dot{G}_{2233}^{s}, \\
& g_{31}=-\dot{G}_{3311}^{s}, \quad g_{44}=-\dot{G}_{2323}^{s}, \quad g_{55}=-\dot{G}_{3131}^{s}, \quad g_{66}=-\dot{G}_{1212}^{s} .
\end{aligned}
$$


Then the strong ellipticity condition (4.1) is equivalent with the following conditions (23]):

$$
\begin{aligned}
& g_{11}>0, \quad g_{22}>0, \quad g_{33}>0, \quad g_{44}>0, \quad g_{55}>0, \quad g_{66}>0, \\
& -2 g_{66}+\kappa_{3}^{i} \sqrt{g_{11} g_{22}}<g_{12}<\kappa_{3}^{s} \sqrt{g_{11} g_{22}}, \\
& -2 g_{44}+\kappa_{1}^{i} \sqrt{g_{22} g_{33}}<g_{23}<\kappa_{1}^{s} \sqrt{g_{22} g_{33}}, \\
& -2 g_{55}+\kappa_{2}^{i} \sqrt{g_{11} g_{33}}<g_{13}<\kappa_{2}^{s} \sqrt{g_{11} g_{33}},
\end{aligned}
$$

where $\left(\kappa_{1}^{i}, \kappa_{1}^{s}\right),\left(\kappa_{2}^{i}, \kappa_{2}^{s}\right)$ and $\left(\kappa_{3}^{i}, \kappa_{3}^{s}\right)$ are solutions with respect to $x, y$ and $z$ of the equation $S(x, y, z) \equiv x^{2}+y^{2}+z^{2}-2 x y z-1=0$, satisfying $|x|<1,|y|<1,|z|<1$ and

$x \in\left\{\frac{g_{23}}{\sqrt{g_{22} g_{33}}}, \frac{g_{23}+2 g_{44}}{\sqrt{g_{22} g_{33}}}\right\}, y \in\left\{\frac{g_{13}}{\sqrt{g_{11} g_{33}}}, \frac{g_{13}+2 g_{55}}{\sqrt{g_{11} g_{33}}}\right\}, z \in\left\{\frac{g_{12}}{\sqrt{g_{11} g_{22}}}, \frac{g_{12}+2 g_{66}}{\sqrt{g_{11} g_{22}}}\right\}$.

We introduce the following function:

$$
\begin{aligned}
& K_{\kappa}\left(x_{3}\right)=-\int_{D\left(x_{3}\right)}\left\{i \left[\left(c_{13}+c_{55}\right)\left(v_{3} \bar{v}_{1,1}-\bar{v}_{3} v_{1,1}\right)+\left(c_{23}+c_{44}\right)\left(v_{3} \bar{v}_{2,2}-\bar{v}_{3} v_{2,2}\right)\right.\right. \\
& \left.\quad+c_{55}\left(v_{1} \bar{v}_{1,3}-\bar{v}_{1} v_{1,3}\right)+c_{44}\left(v_{2} \bar{v}_{2,3}-\bar{v}_{2} v_{2,3}\right)+c_{33}\left(v_{3} \bar{v}_{3,3}-\bar{v}_{3} v_{3,3}\right)\right] \\
& \quad+v_{1}\left[g_{55} \bar{v}_{1,3}+\left(g_{55}-\kappa_{2}\right) \bar{v}_{3,1}\right]+\bar{v}_{1}\left[g_{55} v_{1,3}+\left(g_{55}-\kappa_{2}\right) v_{3,1}\right] \\
& \quad+v_{2}\left[g_{44} \bar{v}_{2,3}+\left(g_{44}-\kappa_{1}\right) \bar{v}_{3,2}\right]+\bar{v}_{2}\left[g_{44} v_{2,3}+\left(g_{55}-\kappa_{1}\right) v_{3,2}\right] \\
& \quad+v_{3}\left[g_{33} \bar{v}_{3,3}+\left(g_{13}+\kappa_{2}\right) \bar{v}_{1,1}+\left(g_{23}+\kappa_{1}\right) \bar{v}_{2,2}\right] \\
& \left.\quad+\bar{v}_{3}\left[g_{33} v_{3,3}+\left(g_{13}+\kappa_{2}\right) v_{1,1}+\left(g_{23}+\kappa_{1}\right) v_{2,2}\right]\right\} d A
\end{aligned}
$$

where $\kappa_{1} \in\left[0,2 g_{44}\right], \kappa_{2} \in\left[0,2 g_{55}\right]$ and $\kappa_{3} \in\left[0,2 g_{66}\right]$. Further, we note that the direct differentiation and basic equations (1.12) imply

$$
\begin{aligned}
& -\frac{d K_{\kappa}}{d x_{3}}\left(x_{3}\right)=2 \int_{D\left(x_{3}\right)}\left[g_{11} v_{1,1} \bar{v}_{1,1}+g_{22} v_{2,2} \bar{v}_{2,2}+g_{33} v_{3,3} \bar{v}_{3,3}+\left(g_{12}+\kappa_{3}\right)\left(v_{1,1} \bar{v}_{2,2}\right.\right. \\
& \left.\left.\quad+\bar{v}_{1,1} v_{2,2}\right)+\left(g_{13}+\kappa_{2}\right)\left(v_{1,1} \bar{v}_{3,3}+\bar{v}_{1,1} v_{3,3}\right)+\left(g_{23}+\kappa_{1}\right)\left(v_{2,2} \bar{v}_{3,3}+\bar{v}_{2,2} v_{3,3}\right)\right] d A \\
& \quad+\int_{D\left(x_{3}\right)}\left[g_{66}\left(v_{1,2} \bar{v}_{1,2}+v_{2,1} \bar{v}_{2,1}\right)+\left(g_{66}-\kappa_{3}\right)\left(v_{1,2} \bar{v}_{2,1}+\bar{v}_{1,2} v_{2,1}\right)\right] d A \\
& \quad+\int_{D\left(x_{3}\right)}\left[g_{55}\left(v_{1,3} \bar{v}_{1,3}+v_{3,1} \bar{v}_{3,1}\right)+\left(g_{55}-\kappa_{2}\right)\left(v_{1,3} \bar{v}_{3,1}+\bar{v}_{1,3} v_{3,1}\right)\right] d A \\
& \quad+\int_{D\left(x_{3}\right)}\left[g_{44}\left(v_{3,2} \bar{v}_{3,2}+v_{2,3} \bar{v}_{2,3}\right)+\left(g_{44}-\kappa_{1}\right)\left(v_{2,3} \bar{v}_{3,2}+\bar{v}_{2,3} v_{3,2}\right)\right] d A .
\end{aligned}
$$

We now choose $\kappa_{1} \in\left[0,2 g_{44}\right], \kappa_{2} \in\left[0,2 g_{55}\right]$ and $\kappa_{3} \in\left[0,2 g_{66}\right]$ so that $P(x, y, z)$, with coordinates $x=\frac{g_{23}+\kappa_{1}}{\sqrt{g_{22} g_{33}}}, y=\frac{g_{13}+\kappa_{2}}{\sqrt{g_{11} g_{33}}}, z=\frac{g_{12}+\kappa_{3}}{\sqrt{g_{11} g_{22}}}$, lies inside the region limited by the 
surface $S(x, y, z)$. On this basis we deduce that

$$
\begin{aligned}
& g_{44}\left(v_{3,2} \bar{v}_{3,2}+v_{2,3} \bar{v}_{2,3}\right)+\left(g_{44}-\kappa_{1}\right)\left(v_{3,2} \bar{v}_{2,3}+\bar{v}_{3,2} v_{2,3}\right) \\
& \quad \geq m_{1}\left(v_{3,2} \bar{v}_{3,2}+v_{2,3} \bar{v}_{2,3}\right), \\
& g_{55}\left(v_{1,3} \bar{v}_{1,3}+v_{3,1} \bar{v}_{3,1}\right)+\left(g_{55}-\kappa_{2}\right)\left(v_{1,3} \bar{v}_{3,1}+\bar{v}_{1,3} v_{3,1}\right) \\
& \quad \geq m_{2}\left(v_{1,3} \bar{v}_{1,3}+v_{3,1} \bar{v}_{3,1}\right), \\
& g_{66}\left(v_{1,2} \bar{v}_{1,2}+v_{2,1} \bar{v}_{2,1}\right)+\left(g_{66}-\kappa_{3}\right)\left(v_{1,2} \bar{v}_{2,1}+\bar{v}_{1,2} v_{2,1}\right) \\
& \quad \geq m_{3}\left(v_{1,2} \bar{v}_{1,2}+v_{2,1} \bar{v}_{2,1}\right), \\
& g_{11} v_{1,1} \bar{v}_{1,1}+g_{22} v_{2,2} \bar{v}_{2,2}+g_{33} v_{3,3} \bar{v}_{3,3}+\left(g_{12}+\kappa_{3}\right)\left(v_{1,1} \bar{v}_{2,2}+\bar{v}_{1,1} v_{2,2}\right) \\
& \quad+\left(g_{13}+\kappa_{2}\right)\left(v_{1,1} \bar{v}_{3,3}+\bar{v}_{1,1} v_{3,3}\right)+\left(g_{23}+\kappa_{1}\right)\left(v_{2,2} \bar{v}_{3,3}+\bar{v}_{2,2} v_{3,3}\right) \\
& \quad \geq m_{4}\left(v_{1,1} \bar{v}_{1,1}+v_{2,2} \bar{v}_{2,2}+v_{3,3} \bar{v}_{3,3}\right),
\end{aligned}
$$

where

$$
m_{1}=\min \left(2 g_{44}-\kappa_{1}, \kappa_{1}\right), m_{2}=\min \left(2 g_{55}-\kappa_{2}, \kappa_{2}\right), \quad m_{3}=\min \left(2 g_{66}-\kappa_{3}, \kappa_{3}\right),
$$

and $m_{4}$ is the lowest positive eigenvalue of the linear transformation given by the matrix

$$
\left(\begin{array}{ccc}
g_{11} & g_{12}+\kappa_{3} & g_{13}+\kappa_{2} \\
g_{12}+\kappa_{3} & g_{22} & g_{23}+\kappa_{1} \\
g_{13}+\kappa_{2} & g_{23}+\kappa_{1} & g_{33}
\end{array}\right)
$$

Therefore, relations (3.33) to (3.37) imply

$$
\begin{aligned}
& -\frac{d K_{\kappa}}{d x_{3}}\left(x_{3}\right) \geq \int_{D\left(x_{3}\right)}\left[m_{1}\left(v_{3,2} \bar{v}_{3,2}+v_{2,3} \bar{v}_{2,3}\right)+m_{2}\left(v_{1,3} \bar{v}_{1,3}+v_{3,1} \bar{v}_{3,1}\right)\right. \\
& \left.\quad+m_{3}\left(v_{1,2} \bar{v}_{1,2}+v_{2,1} \bar{v}_{2,1}\right)+m_{4}\left(v_{1,1} \bar{v}_{1,1}+v_{2,2} \bar{v}_{2,2}+v_{3,3} \bar{v}_{3,3}\right)\right] d A \geq 0,
\end{aligned}
$$

and hence $K_{\kappa}\left(x_{3}\right)$ appears as a nonincreasing function with respect to $x_{3}$ on $[0, L]$. Moreover, the relations (1.14) and (3.32) imply $K_{\kappa}(L)=0$ and so it follows that $K_{\kappa}\left(x_{3}\right) \geq 0$ for all $x_{3} \in[0, L]$ and $K_{\kappa}\left(x_{3}\right)$ is an acceptable measure of the amplitude in question.

We recall that

$$
\begin{aligned}
& \left|g_{44}-\kappa_{1}\right|<g_{44}, \quad\left|g_{55}-\kappa_{2}\right|<g_{55}, \quad\left|g_{66}-\kappa_{3}\right|<g_{66}, \\
& \left|g_{23}+\kappa_{1}\right|<\sqrt{g_{22} g_{33}}, \quad\left|g_{13}+\kappa_{2}\right|<\sqrt{g_{11} g_{33}}, \quad\left|g_{12}+\kappa_{3}\right|<\sqrt{g_{11} g_{22}}
\end{aligned}
$$


and use the Schwarz and arithmetic-geometric mean inequalities and (3.23) to estimate $K_{\kappa}\left(x_{3}\right)$, so we obtain

$$
\begin{aligned}
& K_{\kappa}\left(x_{3}\right) \leq \frac{1}{\sqrt{\lambda_{0}}}\left[\left|c_{13}+c_{55}\right|+\left|c_{55}\right|+2 g_{55}+\sqrt{g_{11} g_{33}}\right] \int_{D\left(x_{3}\right)} v_{1,1} \bar{v}_{1,1} d A \\
& \quad+\frac{1}{\sqrt{\lambda_{0}}}\left[\left|c_{23}+c_{44}\right|+\left|c_{44}\right|+2 g_{44}+\sqrt{g_{22} g_{33}}\right] \int_{D\left(x_{3}\right)} v_{2,2} \bar{v}_{2,2} d A \\
& \quad+\frac{1}{\sqrt{\lambda_{0}}}\left[\left|c_{55}\right|+2 g_{55}\right] \int_{D\left(x_{3}\right)} v_{1,2} \bar{v}_{1,2} d A+\frac{1}{\sqrt{\lambda_{0}}}\left[\left|c_{44}\right|+2 g_{44}\right] \int_{D\left(x_{3}\right)} v_{2,1} \bar{v}_{2,1} d A \\
& \quad+\frac{1}{\sqrt{\lambda_{0}}}\left[\left|c_{13}+c_{55}\right|+\left|c_{23}+c_{44}\right|+\left|c_{33}\right|+g_{33}+g_{55}+\sqrt{g_{11} g_{33}}\right. \\
& \left.\quad+\sqrt{g_{22} g_{33}}\right] \int_{D\left(x_{3}\right)} v_{3,1} \bar{v}_{3,1} d A+\frac{1}{\sqrt{\lambda_{0}}}\left[\left|c_{13}+c_{55}\right|+\left|c_{23}+c_{44}\right|+\left|c_{33}\right|+g_{33}+g_{44}\right. \\
& \left.\quad+\sqrt{g_{11} g_{33}}+\sqrt{g_{22} g_{33}}\right] \int_{D\left(x_{3}\right)} v_{3,2} \bar{v}_{3,2} d A+\frac{1}{\sqrt{\lambda_{0}}}\left(\left|c_{55}\right|+g_{55}\right) \int_{D\left(x_{3}\right)} v_{1,3} \bar{v}_{1,3} d A \\
& \quad+\frac{1}{\sqrt{\lambda_{0}}}\left(\left|c_{44}\right|+g_{44}\right) \int_{D\left(x_{3}\right)} v_{2,3} \bar{v}_{2,3} d A+\frac{1}{\sqrt{\lambda_{0}}}\left(\left|c_{33}\right|+g_{33}\right) \int_{D\left(x_{3}\right)} v_{3,3} \bar{v}_{3,3} d A .
\end{aligned}
$$

From the relations (3.40) and (3.42) we can obtain a first-order differential inequality such as (3.25), and hence we have a spatial estimate such as (3.28), where now $\eta$ is given by

$$
\begin{aligned}
\eta= & \frac{1}{\sqrt{\lambda_{0}}} \max \left\{\frac{\mu_{1}}{m_{4}}, \frac{\mu_{2}}{m_{4}}, \frac{1}{m_{3}}\left(\left|c_{55}\right|+2 g_{55}\right), \frac{1}{m_{3}}\left(\left|c_{44}\right|+2 g_{44}\right), \frac{1}{m_{2}}\left(\mu_{0}+g_{55}\right),\right. \\
& \left.\frac{1}{m_{1}}\left(\mu_{0}+g_{44}\right), \frac{1}{m_{2}}\left(\left|c_{55}\right|+g_{55}\right), \frac{1}{m_{1}}\left(\left|c_{44}\right|+g_{44}\right), \frac{1}{m_{4}}\left(\left|c_{33}\right|+g_{33}\right)\right\},
\end{aligned}
$$

and

$$
\begin{aligned}
& \mu_{1}=\left|c_{13}+c_{55}\right|+\left|c_{55}\right|+2 g_{55}+\sqrt{g_{11} g_{33}}, \quad \mu_{2}=\left|c_{23}+c_{44}\right|+\left|c_{44}\right|+2 g_{44}+\sqrt{g_{22} g_{33}}, \\
& \mu_{0}=\left|c_{13}+c_{55}\right|+\left|c_{23}+c_{44}\right|+\left|c_{33}\right|+g_{33}+\sqrt{g_{11} g_{33}}+\sqrt{g_{22} g_{33}} .
\end{aligned}
$$

4. Concluding remarks. i) In this paper we have obtained some exponential decay estimates, similar to those of Saint-Venant type, for a right homogeneous viscoelastic cylinder, of finite extent, subjected to boundary data varying harmonically in time on one plane end.

Specifically, the estimate described in Theorem 2.1 holds for every value of the frequency of vibrations and for the class of viscoelastic materials whose relaxation tensor is supposed to be symmetric, sufficiently regular and compatible with thermodynamics. In fact, we used just the property expressing the positive definiteness of the half-range sine Fourier transform of the fourth-order tensor $-\dot{G}_{r l m n}(\cdot)$, while the estimate described in Theorem 3.1 holds for every value of the frequency under the weaker assumption that the fourth-order tensor $-\dot{G}_{r l m n}^{s}(\omega)$ is strongly elliptic. 
A relevant example pointing out the relationship between the assumptions upon the half-range Fourier transform $\dot{G}_{r l m n}^{s}(\omega)$ and the properties of $G_{r l m n}(t)$ has been given in Section 2 when the case of decay exponential memory was considered.

ii) The results may be easily extended to a semi-infinite cylinder, namely the case when $L \rightarrow \infty$. In what follows we exemplify this for the case of the measure $I\left(x_{3}\right)$. Then only two possibilities are possible: a) $I\left(x_{3}\right) \geq 0$ for all $x_{3} \in[0, \infty)$; or b) there exists $x_{3}^{*} \in[0, \infty)$ such that $I\left(x_{3}^{*}\right)<0$.

In case a), since $I(\cdot)$ is nonnegative, we deduce the same differential inequality (2.15) and therefore the estimate (2.20) holds.

Let us consider case b). From (2.6) it follows that

$$
I\left(x_{3}\right)<0, \quad x_{3}^{*} \leq x_{3}<\infty,
$$

so that, on $\left[x_{3}^{*}, \infty\right)$, we must change the sign of $I\left(x_{3}\right)$ in the relation (2.7). Repeating the reasoning presented in Section 2 one deduces the following estimate:

$$
-I\left(x_{3}\right) \geq-I\left(x_{3}^{*}\right) \exp \left(\frac{x_{3}-h-x_{3}^{*}}{\gamma_{m}(h, \omega)}\right)>0, \quad x_{3} \in\left[x_{3}^{*}+h, \infty\right) .
$$

Thus, we have the following Phragmèn-Lindelöf alternative.

THEOREM 4.1. In the context of a semi-infinite viscoelastic cylinder for which $-\dot{G}_{r l m n}^{s}(\omega)$ is positive definite, the following alternative holds: a) either $I(\cdot)$ is a nonnegative function on $[0, \infty)$ which decays spatially faster than the exponential $\exp \left(-\frac{x_{3}-h}{\gamma_{m}(h, \omega)}\right)$; or b) there exists $x_{3}^{*} \in[0, \infty)$ such that $I\left(x_{3}^{*}\right)<0$, and then $-I\left(x_{3}\right)$ grows spatially faster than the exponential $\exp \left(\frac{x_{3}-h-x_{3}^{*}}{\gamma_{m}(h, \omega)}\right)$.

We have to outline that a similar alternative can be established for the measures $J_{\kappa}\left(x_{3}\right)$ and $K_{\kappa}\left(x_{3}\right)$.

\section{REFERENCES}

[1] J. N. Flavin and R. J. Knops, Some spatial decay estimates in continuum dynamics, J. Elasticity, 17 (1987), 249-264. MR888318 (88g:73014)

[2] J. N. Flavin, R. J. Knops and L. E. Payne, Decay estimates for the constrained elastic cylinder of variable cross section, Quart. Appl. Math., 47 (1989), 325-350. MR.998106 (90g:73026)

[3] J. N. Flavin, R. J. Knops and L. E. Payne, Energy bounds in dynamical problems for a semi-infinite elastic beam, In Elasticity: Mathematical methods and applications (G. Eason and R.W. Ogden, eds.) The Ian N. Sneddon 70th birthday volume, Ellis Horwood Limited, Chichester, 1990, pp. 101-112.

[4] R. J. Knops, Spatial decay estimates in the vibrating anisotropic elastic beam, In Waves and Stability in Continuous Media (S. Rionero ed.), World Scientific, Singapore, 1991, pp. 192-203. MR1193270

[5] S. Chiriță, Spatial decay estimates for solutions describing harmonic vibrations in a thermoelastic cylinder, J. Thermal Stresses, 18 (1995), 421-436. MR1423300 (97k:73005)

[6] M. Aron and S. Chiriță, Decay and continuous dependence estimates for harmonic vibrations of micropolar elastic cylinders, Arch. Mech., 49 (1997), 665-675. MR1482613 (98j:73006)

[7] W. A. Day, The thermodynamics of simple materials with fading memory, Springer, Berlin, 1972. MR0366234 (51:2482)

[8] M. J. Leitman and G. M. Fischer, The linear theory of viscoelasticity, Handbuch der Physik, Band VIa/3, Springer, Berlin, 1973.

[9] R. M. Christensen, Theory of viscoelasticity: An introduction, 2, Academic Press, New York, 1982.

[10] M. Fabrizio and A. Morro, Mathematical Problems in Linear Viscoelasticity, Society for Industrial and Applied Mathematics, Philadelphia, PA, 1992. MR.1153021(93a:73034) 
[11] M. Fabrizio and A. Morro, Viscoelastic relaxation functions compatible with thermodynamics, J. Elasticity, 19 (1988), 63-75. MR928727 (89m:73019)

[12] R. A. Toupin, Saint-Venant's Principle, Arch. Rational Mech. Anal., 18 (1965), 83-96. MR0172506 (30:2725)

[13] J. K. Knowles, On Saint-Venant's principle in the two-dimensional linear theory of elasticity, Arch. Rational Mech. Anal., 21 (1966), 1-22. MR0187480 (32:4930)

[14] R. S. Lakes and A. Wineman, On Poisson's ratio in linearly viscoelastic solids, J. Elasticity, 85 (2006), 45-63. MR2254005 (2007e:74015)

[15] R. S. Lakes, Viscoelastic solids, CRC Press, Boca Raton, FL, 1998.

[16] S. Chiriţă, M. Ciarletta and M. Fabrizio, Saint-Venant's principle in linear viscoelasticity, Internat. J. Engrg. Sci., 35 (1997), 1221-1236. MR1488530 (99b:73022)

[17] S. Chiriţă and M. Ciarletta, Time-weighted surface power function method for the study of spatial behaviour in dynamics of continua, Eur. J. Mech., A/Solids, 18 (1999), 915-933. MR 1723228 (2000i:74044)

[18] W. A. Day, Restrictions on relaxation functions in linear viscoelasticity, Quart. J. Mech. Appl. Math., 24 (1971), 487-497. MR0317631 (47:6178)

[19] N. S. Wilkes, Thermodynamic restrictions on viscoelastic materials, Quart. J. Mech. Appl. Math., 30 (1977), 209-221. MR0495550 (58:14220)

[20] M. A. Gurtin, The linear theory of elasticity, Handbuch der Physik, Band VIa/2, Springer, Berlin, 1972.

[21] J. Merodio and R. W. Ogden, A note on strong ellipticity for transversely isotropic linearly elastic solids, Quart. J. Mech. Appl. Math., 56 (2003), 589-591. MR2026873

[22] S. Chiriţă, On the strong ellipticity condition for transversely isotropic linearly elastic solids, An. St. Univ. Iasi, Matematica, 52 (2006), 245-250. MR2341092 (2008f:74010)

[23] S. Chiriţă, A. Danescu and M. Ciarletta, On the strong ellipticity of the anisotropic linearly elastic materials, J. Elasticity, 87 (2007), 1-27. MR2310629 (2007m:74013) 